\title{
Medical nanoparticles for next generation drug delivery to the lungs
}

\author{
Sabine H. van Rijt ${ }^{1}$, Thomas Bein ${ }^{2}$ and Silke Meiners ${ }^{1}$
}

\begin{abstract}
Affiliations:
${ }^{1}$ Comprehensive Pneumology Center (CPC), University Hospital, Ludwig-Maximilians University, Helmholtz Zentrum München, Member of the German Center for Lung Research (DZL), Munich, Germany.

${ }^{2}$ Dept of Chemistry, Center for NanoScience (CeNS), Ludwig-Maximilians University, Munich, Germany.

Correspondence: Sabine H. van Rijt, Comprehensive Pneumology Center, Ludwig-Maximilians University, Helmholtz Zentrum München, Max-Lebsche-Platz 31, 81377 Munich, Germany.

E-mail: sabine.vanrijtahelmholtz-muenchen.de
\end{abstract}

ABSTRACT Respiratory diseases are an increasing burden for the ageing population. Although our understanding of these diseases has improved significantly over the past decades, diagnostic and therapeutic options for treating lung diseases, such as chronic obstructive pulmonary disease, idiopathic pulmonary fibrosis and lung cancer, remain limited. Multidisciplinary approaches that bridge the gap between medicinal and materials sciences will likely contribute to promising new therapeutic and diagnostic solutions. One such multidisciplinary approach is the use of nanoparticles as carriers for the delivery of drugs. The advantages of using nanoparticles to deliver drugs include: increased drug concentration at the disease site; minimised drug degradation and loss; ease of creating inhalable formulations; and the possibility of specific cell targeting. This article gives a brief overview on the emerging field of nanocarriers as drug delivery vehicles for the treatment of lung diseases.

@ERSpublications

This article provides an overview on the emerging field of nanocarriers as drug delivery vehicles for lung therapy http://ow.ly/vkNCx 


\section{Introduction}

The lung is a unique organ for drug delivery. Its large surface area, thin epithelium layer and rich blood supply ensure fast drug absorption. These properties offer not only the possibility to treat lung disease, but also allow for the systemic application of drugs. Levels of metabolism in the lung are much lower compared to the gastrointestinal tract and the liver. Therefore, drugs largely bypass metabolism when directly delivered into the lung. Administration of drugs via the lung is made even more attractive by its noninvasiveness and possibility for self-administration. Even though inhaled drugs have been popular for over 50 years, drawbacks such as difficulties with formulation, administration, dosing, compliance and stability of inhaled drugs have limited their clinical success. Only one inhalable therapeutic protein is currently on the market for the treatment of cystic fibrosis (CF) (pulmozyme; Genentech Inc., San Francisco, CA, USA). Inhalable insulin (exubera; Pfizer, New York, NY, USA) for systemic drug delivery was approved in 2006 but has already been withdrawn from the market due to disappointing sales and adverse effects. Another inhalable insulin product (AFREZZA; MannKind Corporation, Valencia, CA, USA) is currently under review by the US Food and Drug Administration (FDA) [1].

Exactly where it is best to deposit drugs in the lungs is not completely clear and depends on the disease type. In asthma, for example, drug delivery to the upper airways is needed while for alveolar lung diseases, such as chronic obstructive pulmonary disease (COPD) and idiopathic pulmonary fibrosis, it is important to have drug deposition in the deep lung rather than the upper airways. Depositing drugs in the lung sounds straightforward but in reality it's quite challenging, for example, due to the efficient clearance mechanisms of the lung (fig. 1). Inhaled particles can be cleared out of the lungs by two systems. In the conducting zone of the lung, particles can efficiently be removed by moving patches of mucus. This mechanism hinders drug delivery to the underlying epithelium, a difficulty that is worsened in lung diseases such as CF and COPD, which are characterised by increased mucus production and thicker mucus. In the deep lung, the monolayer of the alveolar epithelium is extremely thin and the surface liquid lining the alveoli is much thinner than in the bronchial tree and therefore represents less of a hindrance for particle uptake. However, the air side of the alveolar cells is patrolled by macrophages, which engulf and digest insoluble particles that are deposited there quite rapidly [7].

The deposition and distribution of inhaled particles in the lungs is very complex and depends on many factors, such as breathing rate and lung volume of the individual. Additionally, depending on particle size, air flow and desired location in the lung, particle deposition occurs via several different mechanisms. A detailed summary of this is beyond the scope of this article and has been excellently reviewed elsewhere $[2,3]$. However, many studies suggest that the size of the inhaled particles is an important factor in dictating their distribution and deposition site in the lung. There is some consensus in the literature that particles in the range of $1 \mu \mathrm{m}$ to $5 \mu \mathrm{m}$ are deposited in the deep lungs, while particles $>10 \mu \mathrm{m}$ are generally deposited in the oropharyngeal region $[4,5]$. Only a few studies have examined deposition of nanosized particles in lungs and these data suggest that nanosized particles are deposited to a larger extent and more evenly through the lung compared to micron-sized particles [6].

In the alveolar region, the size of the particles appears to dominate the clearance rate. Although many aspects concerning the interactions of particles with macrophages remain unknown, several studies agree on the following statements. 1) Particles at the nanoscale $(\leqslant 200 \mathrm{~nm})$ can cross the cellular barrier independent of energy and are phagocytosed by macrophages to a minor extent. 2) Micron scale particles between $1 \mu \mathrm{m}$ and $5 \mu \mathrm{m}$ are efficiently taken up by macrophages. 3) Particles $>6 \mu \mathrm{m}$ are generally not phagocytosed but rather exhaled [2]. This suggests that particles that are small enough can evade macrophage clearance when deposited in the deep lung. As a result, nanosized particles have ideal deposition characteristics for delivery to alveolar regions of the lung (fig. 1).

The drawbacks associated with generating inhalable drugs, namely formulation and deposition difficulties, can mostly be circumvented by using nanoparticles as almost any drug can be encapsulated. In addition, formulating drugs in nanosized particles, rather than the currently used micron-sized particles, offers additional benefits. Unlike the micron-sized particles, nanoparticles penetrate the lung more deeply and are able to enter the alveolar region. In addition, as mentioned previously, nanosized particles evade macrophage clearance more easily and penetrate the epithelium. At the same time, nanoparticles can be modified on the surface to increase bioavailability or help in penetrating the mucus layers, as well as targeting functionalities to aid drug delivery (discussed in the following sections).

\section{Nano-sized drug carriers}

Over the past decades, the application of nanotechnology in medicine has received much attention. In particular, the use of nanoparticles as carriers for drug delivery is a rapidly growing field of research. The advantages of using nanoscale drug carriers are numerous and include: increased drug circulation times; 
FIGURE 1 Schematic diagram showing the size-dependent deposition of particles in the lungs [2-6].

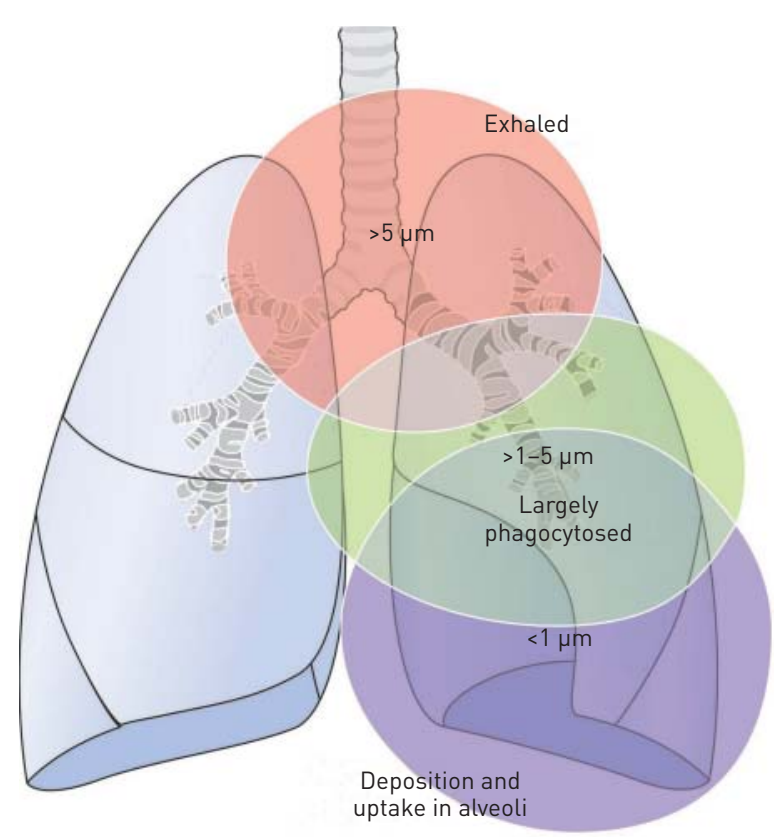

increased drug concentration at the disease site; minimised drug degradation and loss; and ease of administration [8]. It is also important that the use of nanoparticles allows for controlled drug release at the disease site, as well as the possibility for specific cell targeting. Nanocarriers are solid colloidal particles with dimensions usually between $10 \mathrm{~nm}$ and $200 \mathrm{~nm}$. Depending on the nanocarrier, the drug can be captured inside the particle using different techniques and can be retained with different interactions (e.g. covalent and electrostatic interactions). As such not every drug is compatible with every type of nanoparticle. To date, there are many different kinds of known nanoparticles such as polymeric nanoparticles, liposomes, carbon nanotubes, mesoporous silica, protein nanoassemblies, dendrimers, micelles and many more (fig. 2). Depending on the material used and modifications on the surface, nanocarriers can have different properties and drug release characteristics.

Nanoparticles have sizes similar to biological entities such as proteins and viruses (fig. 3 ) and can, therefore, interact with biomolecules on the cell surface and within the cell. Biodegradable polymers have received a lot of attention due to their biocompatibility, possibility for surface modifications and sustained release of the drug [8]. Polymeric particles have also been extensively studied for the delivery of pulmonary medicines; these include asthmatic drugs [9, 10], anti-tuberculosis drugs [11] and anti-cancer drugs [12, 13]. Many types of liposomal nano-formulations have been investigated for their drug delivery properties as well. Currently, there are several US FDA-approved liposomal drug delivery systems on the market and some liposomal drug formulations are in clinical trials [14]. Liposomes seem particularly appropriate for delivery to the lung since they can be prepared from compounds endogenous to the lungs (e.g. lung surfactants). Encouragingly, some liposomal particles made from surfactants (e.g. exosurf (GlaxosSmithKline, Brentford, UK) and alveofact (Lyomark Pharma, Oberhaching, Germany)) are currently on the market for the treatment of acute respiratory distress syndrome [15]. Polymeric and liposomal nanoparticles can be classified as "soft" organic particles. Recently, "hard", often inorganic-based, nanocarriers have received much attention for their drug delivery properties, for example carbon nanotubes and mesoporous silica nanoparticles. Many exciting new nanocarriers with different properties are being reported in this rapidly evolving field of research, making it impossible to highlight all novel findings. However, several properties that are important for all nanocarriers designed for drug delivery are highlighted in figure 4 and discussed in the following sections.

\section{Surface modifications}

Most particles allow for surface modifications that can positively affect their biodistribution and circulation times. Interestingly, nanocarrier hydrophobicity determines, to a large degree, the level of particle binding to blood components. Indeed, nonsurface-modified hydrophobic particles are known to be cleared rapidly [16]. To increase the blood circulation time of these particles, they can be coated with hydrophilic polymers or surfactants. For example, low molecular weight poly(ethylene glycol) (PEG) chains that decorate the particle in a brush-like fashion (PEGylation) are known to reduce phagocytosis [17]. Importantly for chronic obstructive lung diseases, PEGylation of nanoparticles has also been shown to increase mucous 


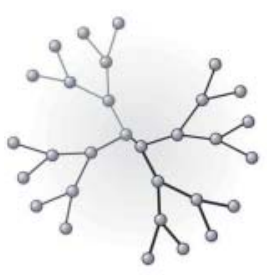

Dendrimer

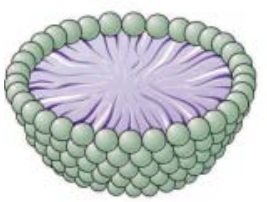

Micelle

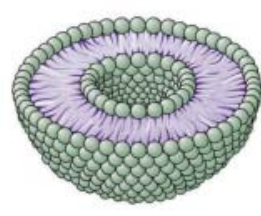

Liposome

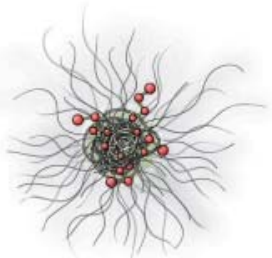

Polymeric nanoparticle

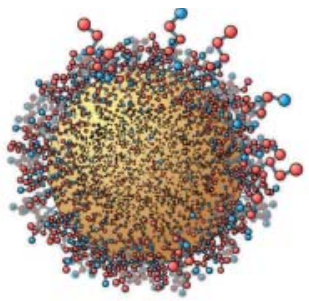

Functionalised gold nanoparticle

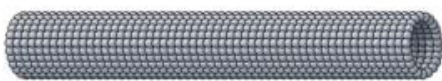

Carbon nanotube

FIGURE 2 Examples of nanoparticles commonly used as drug delivery carriers.

penetration [18]. Chitosan on the other hand is used as surface modification to create more mucoadhesive nanoparticles thus prolonging circulation times [19]. This may be more beneficial for treating nonobstructive lung diseases, such as lung cancer and allergy, since it will allow prolonged residence time at the disease site and thus increased drug uptake and bioavailability. In addition to chemical modifications, the surface of nanoparticles can also be modified in biological fluids by the formation of a so-called protein corona, changing the properties of the particles [20]. Lung surfactant phospholipids are also known to coat inhaled particles [21,22], and this can drastically change their toxicity and cellular uptake [23]. In addition, this may lead to agglomeration [24], which might result in agglomerates that are large enough to be digested by macrophages. The binding strength and mode of binding of lung surfactants to the particles largely depends on the surface of the particle $[25,26]$.

\section{Targeting}

Nanoparticles also offer the possibility for specific tissue or cell targeting, which has the potential to greatly increase their therapeutic effect and decrease drug toxicity. Both active and passive nanoparticle-based targeting is possible. Passive accumulation of nanoparticles in tumour tissue is observed due to a phenomenon known as the enhanced permeability and retention effect. This is caused by the insufficient drainage and poorly regulated angiogenesis of tumours, resulting in an unstructured and leaky vasculature [27]. Passive targeting can also be achieved by surface charge of nanoparticles. For example, cationic

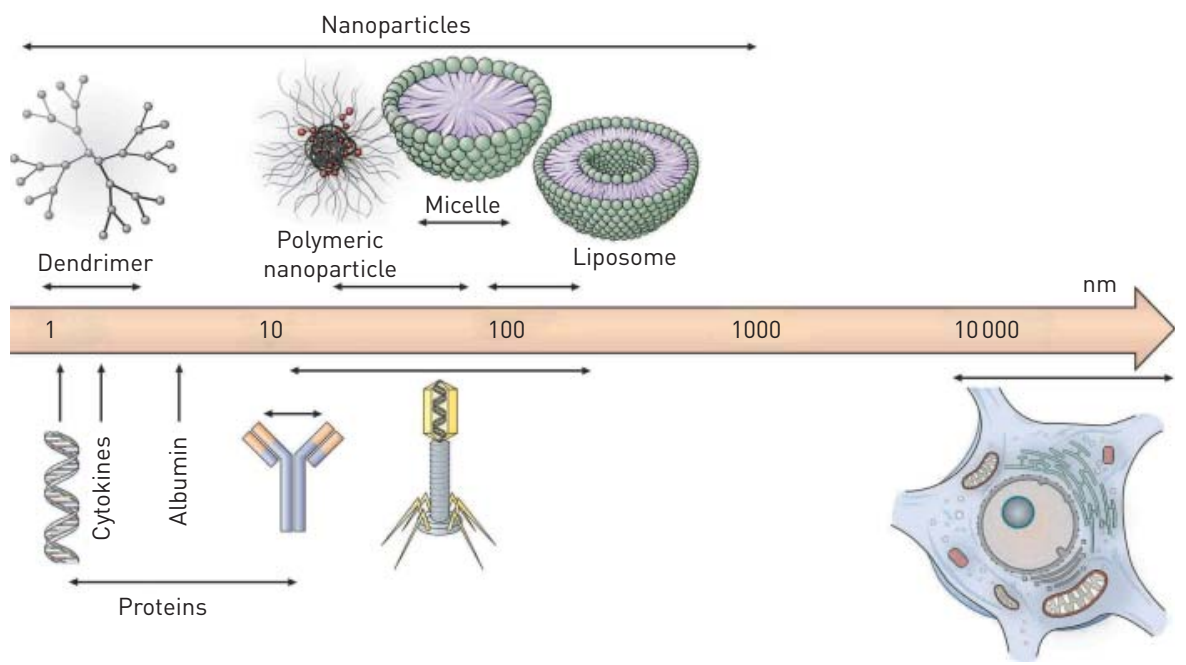

FIGURE 3 Schematic diagram showing the relative sizes of some nanoparticles compared to different biological entities. 


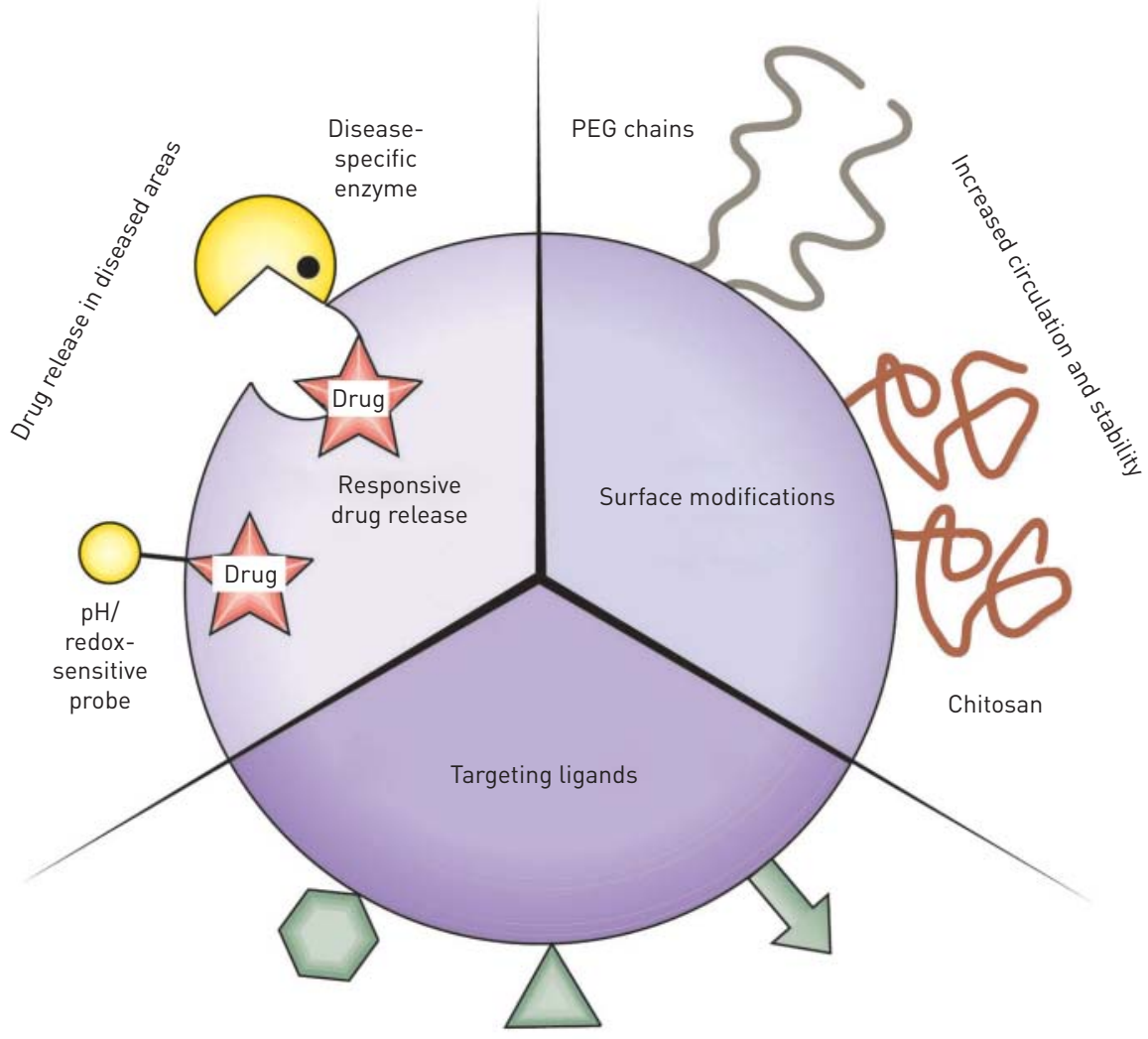

Cell-specific intracellular drug delivery

FIGURE 4 Schematic diagram summarising different properties that are important for the design of nanocarriers for drug delivery. PEG: poly(ethylene glycol).

liposomes loaded with paclitaxel (Bristol-Myers Squibb, New York, NY, USA) were able to target endothelial cells in solid tumours in patients with advanced cancer and liver metastasis [28]. Passive uptake of particles by macrophages can also be used for drug delivery, for example in tuberculosis where the bacteria reside inside the macrophages [29].

Active targeting can be achieved by attaching targeting ligands to the surface of nanocarriers that bind to molecules specific for the diseased cell (i.e. cell receptors that are over-expressed or cell type specific). For example, integrins are transmembrane receptors that mediate interactions with the surrounding extracellular matrix and are upregulated in proliferating tumours cells [30]. Integrin targeting liposomes loaded with doxorubicin inhibited tumour progression in a colon carcinoma mouse model, whereas nontargeting liposomes were ineffective [31]. Several other examples of successful integrin-specific binding of nanoparticles exist [32-34]. Another well-known example for targeted drug delivery is the folate receptor that is over-expressed in a variety of tumours [35]. In one study, folate receptor-targeted polymeric micelles loaded with doxorubicin exhibited increased cellular uptake and circulation time, and decreased cardiotoxicity compared to doxorubicin alone in a nude mice xenograft model [36]. Other examples of cell receptors used for nanoparticle-based active drug targeting include the epidermal growth factor receptor (EGFR) and transferrin receptors [34, 37]. Interestingly, cell type-specific targeting can be achieved by conjugating nanoparticles with cell adhesion antibodies [38].

\section{Controlled drug release}

Controlled release of the drug from the particle is also essential for therapeutic success. Release from the particles can either be stimuli responsive or sustained. Most polymeric and liposome particles have sustained or continuous release of the drug by either diffusion out of the particle or slow degradation of the particle over time. Stimuli-responsive drug release may result in a more targeted release of the drugs and can be achieved by a change in biological environment, such as reducing environment of the cell, change of $\mathrm{pH}$ (e.g. acidic endosomal compartments) or altered levels of disease-specific enzymes. For example, polymeric particles containing $\mathrm{pH}$ sensitive linkers have been used for the removal of the outer polymer coat through 
endocytosis-mediated uptake [39]. The different redox potential between the oxidising extracellular and reducing intracellular environment of tumour cells has also been exploited for polymer biodegradation [40]. Another example of responsive drug release is represented by PEG-peptide-lipid conjugates, where the PEG molecules could be removed from the carriers via cleavage by matrix metalloproteinases that are overexpressed in tumour tissues [41]. Alternatively, external physical stimuli such as light, ultrasound, heat and electric or magnetic fields may also be used to achieve controlled drug release. One example of such an approach is the use of ultrasound-sensitive microbubbles for local release of drugs [42]. Interestingly, microbubbles created from lung surfactants showed a three-fold increase in targeted deposition of the drug compared to common lipid-only microbubbles [43]. Another excellent example is the use of magnetic gradient fields to direct aerosol droplets containing superparamagnetic iron oxide nanoparticles to the desired regions of the lungs in mice [44].

\section{Nanotoxicology}

To fully exploit the potential of nanoparticles for medicinal application, a detailed understanding of biocompatibility, biodistribution and degradation of nanomaterials is required. The physicochemical properties, such as their size, surface charge and shape, will modulate their biological response [45]. For example, rod-shaped nanoparticles are known to be more toxic and harmful than sphere-shaped nanoparticles [46]. In addition, long fibres cannot always be fully engulfed by macrophages, preventing their clearance from the system causing inflammatory responses [47]. Assessing the nanotoxicology of new materials is further complicated by the ability to modify the surface of nanoparticles which affects their bioresponse. For instance, carbon nanotubes are considered to be biopersistant, but it has been shown that functionalised nanotubes can infer water solubility and are rapidly cleared via renal excretion [48]. Due to the infinite possibilities in manipulating particle size, shape and surface chemistry, and because small changes in the physicochemical properties can have a large impact on their biological response, it is necessary to assess the safety of each material separately.

\section{Nanoparticle-based lung therapy}

Lung cancer

Accounting for $>30 \%$ of all cancer deaths, lung cancer is by far the leading cause of cancer deaths in the western world (http://globocan.iarc.fr/). A more tumour-targeted and local delivery of chemotherapeutic drugs to the lungs may improve the outcome and management of this disease. There are many nanoparticle chemotherapeutic formulations under development and several are already on the market. For example abraxane (Abraxis BioScience, Los Angeles, CA, USA), a nano-formulation of paclitaxel and albumin, was shown to be more effective and results in fewer side-effects than using the standard paclitaxel formulation in a phase III study [49]. While mainly used to treat breast cancer, it has recently been approved to treat nonsmall cell lung cancer. Similarly, nano-formulations of docetaxel, a drug commonly used for treatment of nonsmall cell lung cancer, preferentially accumulated in mouse tumour tissue and was more efficient in inhibiting tumour growth and metastatic spread compared to the drug alone or abraxane [50]. Encouragingly, in a recent phase I study, liposomal formulations of cisplatin have successfully been delivered to the lungs via aerosol delivery with higher drug deposition at the tumour site and minimal systemic toxicity [51]. The pulmonary delivery of liposomal 9-nitrocamptothecin, doxorubicin and paxlitaxel has been explored in similar approaches [52-54].

Active targeting strategies for nanoparticle-based drug delivery in the lungs have been limited due to lack of ligands specific to lung cancer cells. Encouragingly, several reports on aptamers (molecules created by selection from a large random sequence pool that bind to a specific target molecule), which can recognise specific small cell lung cancer cell-surface molecular markers with high affinity and specificity, represent a promising strategy to overcome this problem $[55,56]$.

The EGFR has been exploited for targeted delivery to lung cancer cells because it is highly expressed in nonsmall cell lung cancer [34]. For example, gelatin-based nano-formulations with EGFR targeting ligands have been used to target cisplatin to the cancer tissue following inhalation in a subcutaneous mouse model. These EGFR targeting particles were able to reduce tumour volume more effectively, as well as increase the bioavailability of cisplatin in the lung compared to the free drug [57]. Similarly, epidermal growth factor (EGF)-tagged carbon nanotubes containing cisplatin efficiently reduced tumour volume compared to nonEGF labelled tubes [58]. Moreover, liposomes for gene therapy using an anisamide ligand for targeting lung cancer cells overexpressing sigma transmembrane receptors showed therapeutic effectiveness in H1299 lung cancer cells and in in vivo xenograft models [59]. 
Chronic obstructive lung diseases

Mucus hypersecretion and severe inflammation characterise chronic obstructive airway diseases including asthma, CF and COPD. Therefore, the main treatment strategy of these diseases comprises the use of antiinflammatory drugs (e.g. corticosteroids and antibiotics). The use of nanoparticles as drug delivery vehicles in these diseases may prove beneficial as they could provide sustained drug release and overcome airway defences but also target diseased cells or tissues. Indeed, liposomes encapsulating budesonide, a potent corticosteroid, was shown to control the drug release rate and allowed therapeutic concentrations to be maintained in rat lungs for longer periods while reducing systemic toxicity [60]. Notably, nanoparticles also allow for combination treatment of drugs. For example, liposomes containing beclomethasone (glucocorticoid steroid) and formoterol ( $\beta_{2}$-selective receptor agonist) showed that beclomethasone maintained its long-lasting effect while formoterol enhanced lung function and peripheral lung deposition without a significant effect on the mucociliary escalator [61, 62].

The mucus in obstructive airway diseases is not only much thicker, but is also much less viscoelastic. A major additional challenge of using nanoparticles for treating chronic obstructive lung diseases is the penetration of the mucus layer. Studies on that subject suggest that particles with a charged surface are transported across the mucus barrier significantly less [63]. Apart from charge, size also seems to be important for mucociliary clearance of particles. A study by RYTTING et al. [64] showed that small polystyrene particles (diameter $\sim 120 \mathrm{~nm}$ ) moved efficiently through the sputum of CF patients while larger particles (diameter $270-560 \mathrm{~nm}$ ) had significantly less mobility. In addition to size, surface modifications such as PEGylation, rendering the particles more neutrally charged, have been shown to enhance transport across the mucosal barrier [65], and also improve bronchial clearance of particles [63, 66]. Particle uptake and localisation is also dependent on disease type. Inhaled gold particles were taken up less by surface macrophages but at a higher rate by alveolar type I epithelial cells in Scnnlb-transgenic COPD mice compared to wild-type mice [67].

Inhaled steroids are the treatment of choice for controlling asthma, a major public health burden, but their pharmacological effect is relatively short. Encouragingly, polymeric steroid nano-formulations have shown to accumulate and have higher benefits at the site of airway inflammation compared to free steroids [68]. Similarly, polymeric micelles containing budesonide were able to significantly reduce inflammatory cell counts in bronchoalveolar lavage fluid in an asthmatic/COPD rat model [69]. Another approach was reported by JOSHI and MisRa [70] who used liposomes to effectively deliver ketofin fumarate (a mast cell stabiliser known to control the inflammatory response in asthma) to the desired locations in rat lungs using dry powder inhalers [71].

$\mathrm{CF}$ is a monogenic disease characterised by mutation(s) in the CF transmembrane conductance regulator (CFTR) gene that causes exaggerated inflammatory response and decreased mucociliary clearance. Due to this inability to clear mucus and consequently also bacteria and fungi, CF lungs are very susceptible to infections. Therefore, the targeted delivery of antimicrobials by inhalation has received much interest in the past years. Indeed, some liposomal formulations of antibiotics for aerosolised delivery to the lungs are currently in phase II clinical trials [72, 73]. In particular, the liposomal amikacin (Bristol-Myers Squibb, New York, NY, USA) for inhalation therapy showed sustained improvement in lung function and significant reduction of bacterial density in CF patients with chronic pseudomonas lung infections [74]. Similarly, the antifungal drug amphotericin B formulated in liposomes showed reduced side-effects and increased pulmonary deposition and retention in animal models of aspergillosis compared to nonliposomal amphotericin B [75], and was well tolerated in immunocompromised patients [76]. In another approach by VIJ et al. [77], a polymer-based nano-delivery system showed delivery of a proteasome inhibitor to the lungs of mice in a controlled and sustained manner, which could rescue Pseudomonas aeruginosa-lipopolysaccharide induced CF lung disease.

In addition to anti-inflammatory drugs to combat chronic obstructive lung diseases, another promising approach using nanoparticles is gene therapy. Indeed, some nanoparticle vectors could achieve significant levels of transgene expression in the lungs [78]. Several reports have been published on developing cationic liposomes for pulmonary gene delivery [79]. Interestingly, liposomes conjugated to cell-penetrating peptides (e.g. antennapedia octargenine) increased cellular uptake of liposomes to airway cells [80]. Since CF is a monogenic disease, gene therapy seems to be a particularly attractive approach to treat CF [81]. This approach has already shown promise with adenoviruses that could partial correct CF in clinical studies [82]. Unfortunately, transgene expression subsided within weeks and repeating doses triggered adverse immune response. Some clinical trials with cationic lipid formulations have also been studied for their CFTR gene transfer efficiency, but no CF improvement could be observed [83]. $\alpha_{1}$-Antitrypsin deficiency is another monogenic lung disease that would be a good candidate for nanoparticle-based gene therapy [84]. 


\section{Concluding remarks}

Nanocarriers are able to improve efficacy and reduce systemic toxicity for a wide range of drugs. In recent years many advances in this field have allowed for the creation of numerous novel nanoparticles using new carriers, constituents and surface modifications. The use of targeting ligands allows these particles to gain additional abilities creating "smart" multifunctional drug carriers. However, there are some hurdles that this technology faces, which need to be addressed before it can realise its full clinical potential. In addition, the cost/benefit ratio needs to be considered when translating these "smart" nanoparticles to the clinic. For example, the identification of more targets and targeting ligands is required. Nanoparticle toxicity, their effect on the immune system and interaction of the particles with human tissue and fluids needs to be fully understood. In addition to this, for lung therapy, knowledge regarding lung clearance mechanisms and lung deposition of nanoparticles needs to be further developed. To overcome these hurdles and to fully realise the potential of this technology, interactions between scientists from medical, biological and chemical research areas are vital.

\section{References}

1 Neumiller JJ, Campbell RK. Technosphere insulin: an inhaled prandial insulin product. BioDrugs 2010; 24: $165-172$.

2 Carvalho TC, Peters JI, Williams RO 3rd. Influence of particle size on regional lung deposition - what evidence is there? Int J Pharm 2011; 406: 1-10.

3 Smola M, Vandamme T, Sokolowski A. Nanocarriers as pulmonary drug delivery systems to treat and to diagnose respiratory and non respiratory diseases. Int J Nanomed 2008; 3: 1-19.

4 Malcolmson RJ, Embleton JK. Dry powder formulations for pulmonary delivery. Pharm Sci Technol Today 1998; 1: 394-398.

5 Chow AH, Tong HH, Chattopadhyay P, et al. Particle engineering for pulmonary drug delivery. Pharm Res 2007; 24: 411-437.

6 Bhavna, Ahmad FJ, Mittal G, et al. Nano-salbutamol dry powder inhalation: a new approach for treating bronchoconstrictive conditions. Eur J Pharm Biopharm 2009; 71: 282-291.

7 Folkesson HG, Matthay MA, Westrom BR, et al. Alveolar epithelial clearance of protein. J Appl Physiol 1996; 80: 1431-1445.

8 Schutz CA, Juillerat-Jeanneret L, Mueller $\mathrm{H}$, et al. Therapeutic nanoparticles in clinics and under clinical evaluation. Nanomed (Lond) 2013; 8: 449-467.

9 Wernig K, Griesbacher M, Andreae F, et al. Depot formulation of vasoactive intestinal peptide by protamine-based biodegradable nanoparticles. J Control Release 2008; 130: 192-198.

10 Oh YJ, Lee J, Seo JY, et al. Preparation of budesonide-loaded porous PLGA microparticles and their therapeutic efficacy in a murine asthma model. J Control Release 2011; 150: 56-62.

11 Ahmad Z, Sharma S, Khuller GK. Inhalable alginate nanoparticles as antitubercular drug carriers against experimental tuberculosis. Int J Antimicrob Agents 2005; 26: 298-303.

12 Azarmi S, Tao X, Chen $\mathrm{H}$, et al. Formulation and cytotoxicity of doxorubicin nanoparticles carried by dry powder aerosol particles. Int J Pharm 2006; 319: 155-161.

13 Karra N, Nassar T, Ripin AN, et al. Antibody conjugated PLGA nanoparticles for targeted delivery of paclitaxel palmitate: efficacy and biofate in a lung cancer mouse model. Small 2013; 9: 4221-4236.

14 Zhang L, Gu FX, Chan JM, et al. Nanoparticles in medicine: therapeutic applications and developments. Clin Pharmacol Ther 2008; 83: 761-769.

15 Muller RH, Mader K, Gohla S. Solid lipid nanoparticles (SLN) for controlled drug delivery - a review of the state of the art. Eur J Pharm Biopharm 2000; 50: 161-177.

16 Yoo JW, Doshi N, Mitragotri S. Adaptive micro and nanoparticles: temporal control over carrier properties to facilitate drug delivery. Adv Drug Deliver Rev 2011; 63: 1247-1256.

17 Amoozgar Z, Yeo Y. Recent advances in stealth coating of nanoparticle drug delivery systems. Wiley Interdiscip Rev Nanomed Nanobiotechnol 2012; 4: 219-233.

18 Yuan H, Chen CY, Chai GH, et al. Improved transport and absorption through gastrointestinal tract by PEGylated solid lipid nanoparticles. Mol Pharm 2013; 10: 1865-1873.

19 Doh KO, Yeo Y. Application of polysaccharides for surface modification of nanomedicines. Ther Deliv 2012; 3: 1447-1456.

20 Monopoli MP, Aberg C, Salvati A, et al. Biomolecular coronas provide the biological identity of nanosized materials. Nat Nanotechnol 2012; 7: 779-786.

21 Ruge CA, Schaefer UF, Herrmann J, et al. The interplay of lung surfactant proteins and lipids assimilates the macrophage clearance of nanoparticles. PLos One 2012; 7: e40775.

22 Ruge CA, Kirch J, Canadas O, et al. Uptake of nanoparticles by alveolar macrophages is triggered by surfactant protein A. Nanomedicine 2011; 7: 690-693.

23 Schleh C, Hohlfeld JM. Interaction of nanoparticles with the pulmonary surfactant system. Inhal Toxicol 2009; 21 : Suppl. 1, 97-103.

24 Kendall M, Ding P, Mackay RM, et al. Surfactant protein D (SP-D) alters cellular uptake of particles and nanoparticles. Nanotoxicology 2013; 7: 963-973.

25 Kumar P, Bohidar HB. Interaction of soot derived multi-carbon nanoparticles with lung surfactants and their possible internalization inside alveolar cavity. Indian J Exp Biol 2010; 48: 1037-1042.

26 Wang L, Castranova V, Mishra A, et al. Dispersion of single-walled carbon nanotubes by a natural lung surfactant for pulmonary in vitro and in vivo toxicity studies. Part Fibre Toxicol 2010; 7: 31.

27 Hashizume H, Baluk P, Morikawa S, et al. Openings between defective endothelial cells explain tumor vessel leakiness. Am J Pathol 2000; 156: 1363-1380. 
28 Fasol U, Frost A, Buchert M, et al. Vascular and pharmacokinetic effects of EndoTAG-1 in patients with advanced cancer and liver metastasis. Ann Oncol 2012; 23: 1030-1036.

29 Hirota K, Hasegawa T, Hinata H, et al. Optimum conditions for efficient phagocytosis of rifampicin-loaded PLGA microspheres by alveolar macrophages. J Control Release 2007; 119: 69-76.

30 Marelli UK, Rechenmacher F, Sobahi TR, et al. Tumor targeting via integrin ligands. Front Oncol 2013; 3: 222.

31 Schiffelers RM, Koning GA, ten Hagen TL, et al. Anti-tumor efficacy of tumor vasculature-targeted liposomal doxorubicin. J Control Release 2003; 91: 115-122.

32 Kluza E, van der Schaft DW, Hautvast PA, et al. Synergistic targeting of $\alpha_{v} \beta_{3}$ integrin and galectin-1 with heteromultivalent paramagnetic liposomes for combined MR imaging and treatment of angiogenesis. Nano Lett 2010; 10: 52-58.

33 Chen $\mathrm{K}$, Xie J, Xu H, et al. Triblock copolymer coated iron oxide nanoparticle conjugate for tumor integrin targeting. Biomaterials 2009; 30: 6912-6919.

34 Mehra NK, Mishra V, Jain NK. Receptor-based targeting of therapeutics. Ther Deliv 2013; 4: 369-394.

35 Sudimack J, Lee RJ. Targeted drug delivery via the folate receptor. Adv Drug Deliv Rev 2000; 41: 147-162.

36 Yoo HS, Park TG. Folate receptor targeted biodegradable polymeric doxorubicin micelles. J Control Release 2004; 96: 273-283.

37 Daniels TR, Bernabeu E, Rodriguez JA, et al. The transferrin receptor and the targeted delivery of therapeutic agents against cancer. Biochim Biophys Acta 2012; 1820: 291-317.

38 Wiewrodt R, Thomas AP, Cipelletti L, et al. Size-dependent intracellular immunotargeting of therapeutic cargoes into endothelial cells. Blood 2002; 99: 912-922.

39 Meyer M, Wagner E. pH-responsive shielding of non-viral gene vectors. Expert Opin Drug Deliv 2006; 3: 563-571.

40 Meyer M, Dohmen C, Philipp A, et al. Synthesis and biological evaluation of a bioresponsive and endosomolytic siRNA-polymer conjugate. Mol Pharm 2009; 6: 752-762.

41 Hatakeyama H, Akita H, Kogure K, et al. Development of a novel systemic gene delivery system for cancer therapy with a tumor-specific cleavable PEG-lipid. Gene Ther 2007; 14: 68-77.

42 Geers B, Dewitte H, De Smedt SC, et al. Crucial factors and emerging concepts in ultrasound-triggered drug delivery. J Control Release 2012; 164: 248-255.

43 Sirsi SR, Fung C, Garg S, et al. Lung surfactant microbubbles increase lipophilic drug payload for ultrasoundtargeted delivery. Theranostics 2013; 3: 409-419.

44 Hasenpusch G, Geiger J, Wagner K, et al. Magnetized aerosols comprising superparamagnetic iron oxide nanoparticles improve targeted drug and gene delivery to the lung. Pharm Res 2012; 29: 1308-1318.

Thorley AJ, Tetley TD. New perspectives in nanomedicine. Pharmacol Ther 2013; 140: 176-185.

Sharifi S, Behzadi S, Laurent S, et al. Toxicity of nanomaterials. Chem Soc Rev 2012; 41: 2323-2343.

Madani SY, Mandel A, Seifalian AM. A concise review of carbon nanotube's toxicology. Nano Rev 2013 (in press DOI: $10.3402 /$ nano.v4i0.21521).

48 Singh R, Pantarotto D, Lacerda L, et al. Tissue biodistribution and blood clearance rates of intravenously administered carbon nanotube radiotracers. Proc Natl Acad Sci USA 2006; 103: 3357-3362.

49 Gradishar WJ, Tjulandin S, Davidson N, et al. Phase III trial of nanoparticle albumin-bound paclitaxel compared with polyethylated castor oil-based paclitaxel in women with breast cancer. J Clin Oncol 2005; 23: 7794-7803.

50 Ernsting MJ, Murakami M, Undzys E, et al. A docetaxel-carboxymethylcellulose nanoparticle outperforms the approved taxane nanoformulation, Abraxane, in mouse tumor models with significant control of metastases. J Control Release 2012; 162: 575-581.

51 Wittgen BP, Kunst PW, van der Born K, et al. Phase I study of aerosolized SLIT cisplatin in the treatment of patients with carcinoma of the lung. Clin Cancer Res 2007; 13: 2414-2421.

52 Latimer P, Menchaca M, Snyder RM, et al. Aerosol delivery of liposomal formulated paclitaxel and vitamin E analog reduces murine mammary tumor burden and metastases. Exp Biol Med 2009; 234: 1244-1252.

53 Verschraegen CF, Gilbert BE, Loyer E, et al. Clinical evaluation of the delivery and safety of aerosolized liposomal 9nitro-20(s)-camptothecin in patients with advanced pulmonary malignancies. Clin Cancer Res 2004; 10: 2319-2326.

54 Otterson GA, Villalona-Calero MA, Hicks W, et al. Phase I/II study of inhaled doxorubicin combined with platinum-based therapy for advanced non-small cell lung cancer. Clin Cancer Res 2010; 16: 2466-2473.

55 Chen HW, Medley CD, Sefah K, et al. Molecular recognition of small-cell lung cancer cells using aptamers. ChemMedChem 2008; 3: 991-1001.

56 Zhao Z, Xu L, Shi X, et al. Recognition of subtype non-small cell lung cancer by DNA aptamers selected from living cells. Analyst 2009; 134: 1808-1814.

57 Tseng CL, Su WY, Yen KC, et al. The use of biotinylated-EGF-modified gelatin nanoparticle carrier to enhance cisplatin accumulation in cancerous lungs via inhalation. Biomaterials 2009; 30: 3476-3485.

58 Bhirde AA, Patel S, Sousa AA, et al. Distribution and clearance of PEG-single-walled carbon nanotube cancer drug delivery vehicles in mice. Nanomed (Lond) 2010; 5: 1535-1546.

59 Li SD, Huang L. Targeted delivery of antisense oligodeoxynucleotide and small interference RNA into lung cancer cells. Mol Pharm 2006; 3: 579-588.

60 Joshi M, Misra AN. Pulmonary disposition of budesonide from liposomal dry powder inhaler. Methods Find Exp Clin Pharmacol 2001; 23: 531-536.

61 Saari SM, Vidgren MT, Herrala J, et al. Possibilities of formoterol to enhance the peripheral lung deposition of the inhaled liposome corticosteroids. Resp Med 2002; 96: 999-1005.

62 Saari M, Vidgren MT, Koskinen MO, et al. Pulmonary distribution and clearance of two beclomethasone liposome formulations in healthy volunteers. Int J Pharm 1999; 181: 1-9.

63 Suk JS, Lai SK, Boylan NJ, et al. Rapid transport of muco-inert nanoparticles in cystic fibrosis sputum treated with N-acetyl cysteine. Nanomed (Lond) 2011; 6: 365-375.

64 Rytting E, Nguyen J, Wang X, et al. Biodegradable polymeric nanocarriers for pulmonary drug delivery. Expert Opin Drug Deliv 2008; 5: 629-639.

65 Sanders N, Rudolph C, Braeckmans K, et al. Extracellular barriers in respiratory gene therapy. Adv Drug Deliver Rev 2009; 61: 115-127.

66 Lai SK, Wang YY, Hanes J. Mucus-penetrating nanoparticles for drug and gene delivery to mucosal tissues. Adv Drug Deliver Rev 2009; 61: 158-171. 
Geiser M, Quaile O, Wenk A, et al. Cellular uptake and localization of inhaled gold nanoparticles in lungs of mice with chronic obstructive pulmonary disease. Part Fibre Toxicol 2013; 10: 19.

68 Matsuo Y, Ishihara T, Ishizaki J, et al. Effect of betamethasone phosphate loaded polymeric nanoparticles on a murine asthma model. Cell Immunol 2009; 260: 33-38.

69 Sahib MN, Darwis Y, Peh KK, et al. Rehydrated sterically stabilized phospholipid nanomicelles of budesonide for nebulization: physicochemical characterization and in vitro, in vivo evaluations. Int J Nanomedicine 2011; 6: 2351-2366.

70 Joshi M, Misra A. Dry powder inhalation of liposomal ketotifen fumarate: formulation and characterization. Int J Pharm 2001; 223: 15-27.

71 Joshi M, Misra A. Disposition kinetics of ketotifen from liposomal dry powder for inhalation in rat lung. Clin Exp Pharmacol Physiol 2003; 30: 153-156.

72 Bruinenberg P, Serisier D, Cipolla D, et al. Safety, tolerability, pharmacokinetics and antimicrobial activity of inhaled liposomal ciprofloxacin formulations in humans. Pediatr Pulm 2010; 354-354.

73 Weers J, Metzheiser B, Taylor G, et al. A gamma scintigraphy study to investigate lung deposition and clearance of inhaled amikacin-loaded liposomes in healthy male volunteers. J Aerosol Med Pulm Drug Deliv 2009; 22: 131-138.

74 Okusanya OO, Bhavnani SM, Hammel J, et al. Pharmacokinetic and pharmacodynamic evaluation of liposomal amikacin for inhalation in cystic fibrosis patients with chronic pseudomonal infection. Antimicrob Agents Chemother 2009; 53: 3847-3854.

75 Allen SD, Sorensen KN, Nejdl MJ, et al. Prophylactic efficacy of aerosolized liposomal (AmBisome) and nonliposomal (Fungizone) amphotericin B in murine pulmonary aspergillosis. J Antimicrob Chemother 1994; 34: 1001-1013.

76 Slobbe L, Boersma E, Rijnders BJ. Tolerability of prophylactic aerosolized liposomal amphotericin-B and impact on pulmonary function: data from a randomized placebo-controlled trial. Pulm Pharmacol Ther 2008; 21: 855-859.

77 Vij N, Min T, Marasigan R, et al. Development of PEGylated PLGA nanoparticle for controlled and sustained drug delivery in cystic fibrosis. J Nanobiotechnology 2010; 8: 22.

78 Rosenecker J, Huth S, Rudolph C. Gene therapy for cystic fibrosis lung disease: current status and future perspectives. Curr Opin Mol Ther 2006; 8: 439-445.

79 Swaminathan J, Ehrhardt C. Liposomal delivery of proteins and peptides. Expert Opin Drug Deliv 2012; 9: 1489-1503.

80 Cryan SA, Devocelle M, Moran PJ, et al. Increased intracellular targeting to airway cells using octaarginine-coated liposomes: in vitro assessment of their suitability for inhalation. Mol Pharm 2006; 3: 104-112.

81 Mueller C, Flotte TR. Gene therapy for cystic fibrosis. Clin Rev Allergy Immunol 2008; 35: 164-178.

82 Lee TW, Matthews DA, Blair GE. Novel molecular approaches to cystic fibrosis gene therapy. Biochem J 2005; 387 : $1-15$.

83 Zabner J, Cheng SH, Meeker D, et al. Comparison of DNA-lipid complexes and DNA alone for gene transfer to cystic fibrosis airway epithelia in vivo. J Clin Invest 1997; 100: 1529-1537.

84 Brigham KL, Lane KB, Meyrick B, et al. Transfection of nasal mucosa with a normal alphal-antitrypsin gene in alpha ${ }_{1}$-antitrypsin-deficient subjects: comparison with protein therapy. Hum Gene Ther 2000; 11: 1023-1032. 